\title{
Postraumatic Torsion of Wandering Spleen in 9-year-old Boy
}

\author{
Tina Zavidic ${ }^{1,2^{\star}}$, Dino Lovrinic ${ }^{3}$, Marko Bogovic ${ }^{4}$, Branimir Lodeta $^{5}$
}

\author{
${ }^{1}$ Istrian Health Centers, Pazin, \\ Croatia \\ ${ }^{2}$ University of Rijeka, Faculty of \\ Medicine, Rijeka, Croatia \\ ${ }^{3}$ Family Physician office Lovrinic, \\ Pazin, Croatia \\ ${ }^{4}$ Department of Pediatric Surgery, \\ Klinikum Klagenfurt, Klagenfurt, \\ Austria \\ ${ }^{5}$ Department of Urology and \\ Andrology, Klinikum Klagenfurt, \\ Klagenfurt, Austria
}

\section{Correspondence:}

Tina Zavidic

Address: Istria Health Center, Pazin, J. Dobrile 1, 52000 Pazin, Croatia

Email: tina.zavidic@gmail.com

Received: 07.04.2020,

Accepted: 07.05.2020

https://doi.org/10.5799/jcei/8271

\begin{abstract}
Post-traumatic torsion of wondering spleen is extremely rare cause of acute abdominal pain in children with only few cases reported. We report a case of a 9-year-old boy with wandering spleen torsion after bike crash and subacute clinical presentation. After a bicycle crash and a period of persistent fever family physician performed an ultrasound of abdomen which showed a round focal lesion of $10 \times 12 \mathrm{~cm}$ in size, a spleen-like echogenicity in the middlelower abdominal position and in the small pelvis, which raised suspicion of ectopic (wandering) spleen. The abdominal MSCT confirmed the diagnosis of wandering spleen, Numerous immunologic, hematological, radiological, infectious, endocrinological, chromosomal and cytological tests and markers confirmed a diagnosis of the splenic malformation. A surgeon arranged to perform splenopexy after checking the vaccination status. Laparotomy was initiated. The splenic artery and vein, which was in a triple twist, was released and due to the resulting path, the spleen itself was larger than the CT finding. The spleen was placed under the left ribs and splenopexy was performed. At the fifteen-day and two-month follow ups, the patient felt good, was painless, afebrile and the spleen gradually decreased. Wondering spleen should be considered as a possible cause of acute abdominal pain when the spleen is not seen in its usual position. The treatment of choice is surgery, with the goal of preservation of the organ whenever possible.
\end{abstract}

Keywords: wandering spleen, ectopic spleen, splenopexy, torsion

\section{INTRODUCTION}

Wandering spleen is a rare medical entity with incidence less than $0.2 \%$ and represent indication for 2 per 1000 splenectomies [1]. It is characterized by excessive mobility and displacement of the spleen from its normal location in the left hypochondrium. It is most often seen in women in the third decade of life and in young children, especially below one year. The most common presentation in children is acute abdominal pain, but patient can also be asymptomatic [2,3]. Paediatric cases of traumatic torsion are extremely rare [3].

Imaging with ultrasound, computed tomography and magnetic resonance confirms diagnosis - absence of spleen in expected location and soft-tissue mass lower in abdomen or even in pelvis. The treatment of choice is surgery with splenopexy (whenever possible) or splenectomy.

\section{Case}

At the beginning of April 2019, parents brought a nine-year-old boy to a family physician after a bicycle crash. He had numerous minor injuries of the face. At the examination the boy was febrile $\left(38^{\circ} \mathrm{C}\right)$ and complained of sore throat and painful urination. Due to the hyperemic pharynx, the doctor prescribed an antibiotic and indicated laboratory tests which have shown an moderate thrombocytopenia (150 x $\left.10^{9} / \mathrm{L}\right)$, moderate leukopenia $\left(4.3 \times 10^{9} / \mathrm{L}\right)$, low iron level $(3 \mu \mathrm{mol} / \mathrm{L})$, and normal hemoglobin level of $125 \mathrm{~g} / \mathrm{L}$ (Erythrocyte $4,68 \times 10^{12} / \mathrm{L}$, hematocrit $0.388 \%$ ). Inflammatory parameters and urine findings were within normal limits.

Since temperature persisted for 7 days, the family physician decided to do another laboratory control, expanded with mononucleosis and cytomegalovirus markers and performed an ultrasound of 


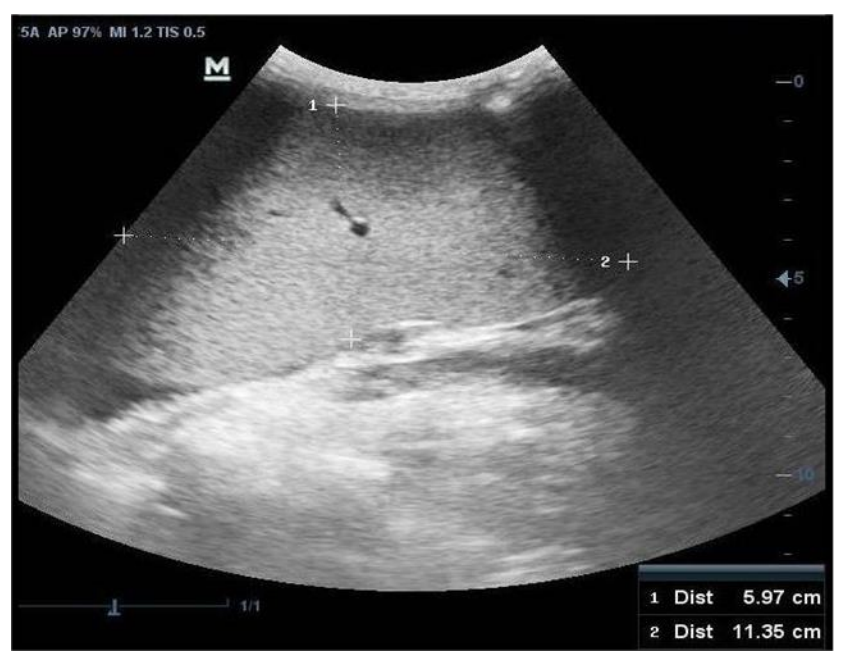

Figure 1. Ultrasound of ectopic spleen in a 9-year-old patient

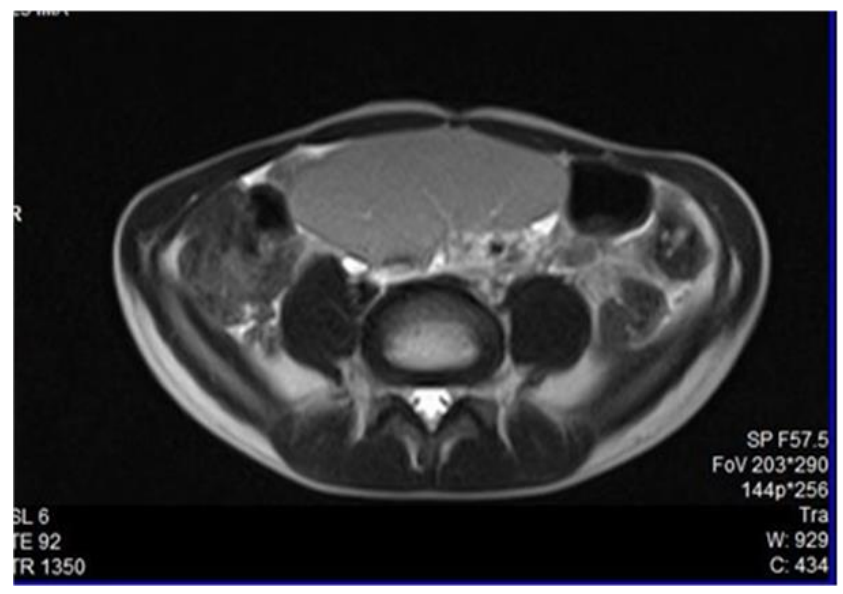

Figure 2. CT view of spleen through the umbilical line

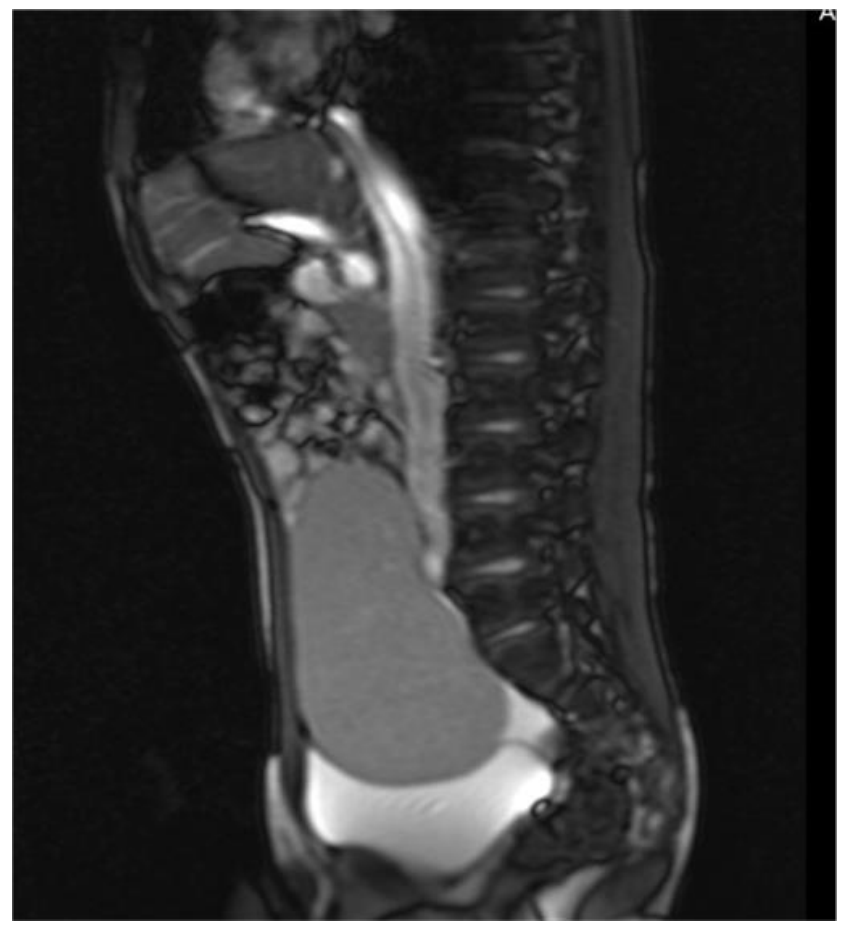

Figure 3. Sagittal CT of abdomen abdomen which showed a round focal lesion of $10 \times 12 \mathrm{~cm}$ in size, a spleen-like echogenicity in the middle-lower abdominal position and in the small pelvis. Color Doppler ultrasound scan showed visible arterial and vein flows. The ultrasound raised suspicion of ectopic (wandering) spleen (Figure 1). Additional laboratory findings indicated the development of anaemia ( $\mathrm{Hb} 112$ ) and further decline in platelet count $\left(143 \times 10^{9} / \mathrm{L}\right)$.

The abdominal MSCT confirmed the diagnosis of wandering spleen, located lower in the abdomen, with bladder impression, 15 x $9.5 \mathrm{~cm}$, physiological signal intensity and homogeneous structure (Figures 2 and 3 ).

The patient was referred to the Clinical Hospital Center where he was hospitalized for two days at the Department of Hematology and Oncology, performing numerous immunologic, hematological, radiological, infectious, endocrinological, chromosomal and cytological tests and markers. He was released home with a diagnosis of the splenic malformation.

Two months after the onset of symptoms, the parents agreed with the family physician to seek a second opinion of a surgeon, who concluded that the child had spleen fixation disorder. The surgeon further arranged to perform splenopexy after checking the vaccination status.

In the following days the boy was vaccinated against streptococcus pneumoniae and meningococcus to prevent overwhelming post-splenectomy infection in case a splenectomy should be needed. He was already regulary Haemophilus influenzae type B vaccinated.

At the beginning of July, an operative procedure was initiated and laparoscopic exploration revealed a clearly visible multiple torsion of splenic artery and vein. Since laparoscopic manipulation was unsafe, the surgeons decided to convert procedure to laparotomy. The splenic artery and vein, which was in a triple twist, was released and due to the resulting path, the spleen itself was larger than the CT finding $(20 \times 10 \mathrm{~cm})$ (Figure 4).

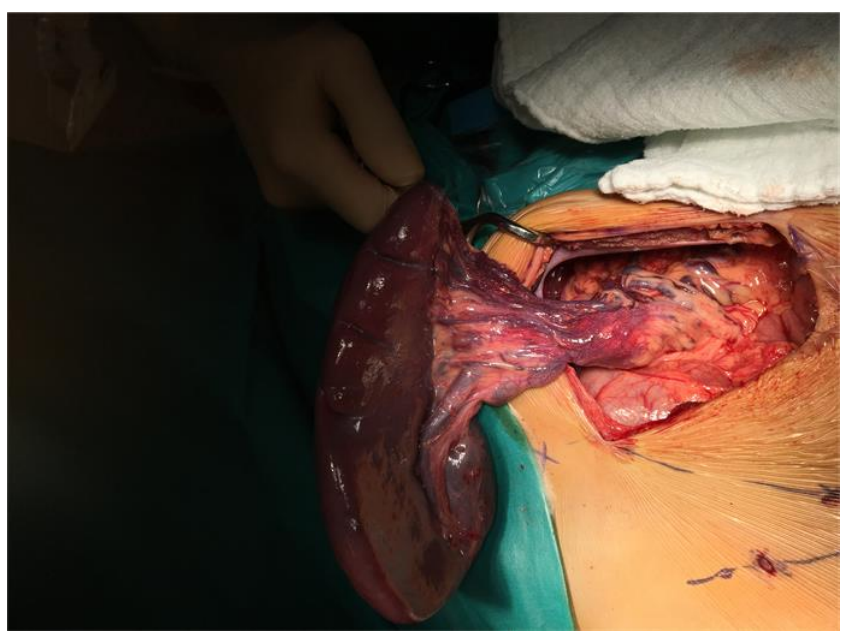

Figure 4. Splenopexy of a nine-year-old boy 
The spleen was placed under the left ribs and splenopexy was performed. By mobilizing the left flexion of the column, a pocket for the lower half of the spleen was created, and with two dictonial patches the spleen was additionally suspended. The left lobe of the liver was also mobilized thus creating a space for the upper half of the spleen. The whole area was additionally covered with omentum.

At the fifteen-day and two-month follow ups, the patient felt good, was painless, afebrile and the spleen gradually decreased. At the last ultrasound examination, spleen size was $12.3 \times 4.5 \mathrm{~cm}$.

\section{DISCUSSION}

Wandering spleen is defined as a condition in which the spleen migrates from its normal position in the left hypochondrium, mostly due to weakness of the primary supporting ligament of spleen with elongation of its vascular pedicle. The clinical spectrum can range from an asymptomatic abdominal mass, an incidental finding on routine abdominal sonography, intermittent abdominal pain and splenomegaly to severe abdominal pain and discomfort due to torsion of the splenic vascular pedicle [4].

Pedicle torsion causes partial and complete spleen infarction, and consequently acute abdomen and represent indication for urgent surgical intervention. Splenic pedicle torsion is diagnosed in about $0.2-0.3 \%$ of patients with splenectomy [5].

Ultrasonography shows the characteristics of the spleenlike mass in ectopic positions in the abdomen and pelvis, and the absence of spleen tissue in the left upper abdomen. CT imaging provides rapid confirmation of diagnosis. The main advantage of magnetic resonance imaging is that ionizing radiation is not used and superior imaging of soft tissues.

The treatment of choice is laparoscopic surgery, with splenopexy whenever possible [6]. Laparoscopic splenopexy in children is feasible, with no reported conversions till now.

In our case, wandering spleen with posttraumatic torsion was misdiagnosed as a splenic malformation. This can be explained by relatively rareness of condition, especially in school-age children, as well as subacute onset of symptoms. Delay in surgical treatment, luckily, did not cause major health issues.

Patients with an absent or dysfunctional spleen are at a life-long increased risk of bacterial infection due to pneumoccocus, meningococcus and Haemophilus spp. [7].

Ideally patients should be vaccinated at least two weeks before procedure (possible splenectomy), as it was done in our patient due to possible need for splenectomy [8].

Post-traumatic torsion of wondering spleen is extremely rare cause of acute abdominal pain in children with only few cases reported [3,9]. Suspicion of wandering spleen should arise when the spleen is not seen in its usual position during imaging. The treatment of choice is laparoscopic splenopexy, with the goal of preservation of the organ whenever possible, but splenectomy is still reserved for those cases of massive infarction or thrombosis of splenic vessels.

\section{CONCLUSION}

Wondering spleen should be considered as a possible cause of acute abdominal pain when the spleen is not seen in its usual position. The treatment of choice is surgery, with the goal of preservation of the organ whenever possible. Preferred treatment is laparoscopic splenopexy, with or without the use of absorbable mesh, but splenectomy is still reserved for those cases of massive infarction or thrombosis of splenic vessels.

Declaration of interest: The authors report no conflicts of interest.

Financial Disclosure: No financial support was received.

\section{REFERENCES}

1. Fujiwara T, Takehara Y, Isoda H, Ichijo K, Tooyama N, Kodaira N, et al. Torsion of the wandering spleen: CT and angiographic appearance. J Comput Assist Tomogr. 1995;19:84-6. doi: 10.1097/00004728-199501000-00016.

2. Blouhos K, Boulas KA, Salpigktidis I, Barettas N, Hatzigeorgiadis A. Ectopic spleen: An easily identifiable but commonly undiagnosed entity until manifestation of complications. Int J Surg Case Rep. 2014; 5: 451-4. doi: 10.1016/j.ijscr.2014.05.010.

3. Saouab R, Dafiri R. Post-traumatic acute abdominal pain. Br J Radiol. 2010; 83: 804-6. doi: 10.1259/bjr/88857252.

4. Puranik AK, Mehra R, Chauhan S, Pandey R. Wandering spleen: a surgical enigma. Gastroenterol Rep. 2015; gov034. doi: 10.1093/gastro/gov034.

5. Mortelé KJ, Mortelé B, Silverman SG. CT features of the accessory spleen. AJR Am J Roentgenol. 2004; 183: 16537. doi: 10.2214/ajr.183.6.01831653.

6. Palanivelu C, Rangarajan M. Mesh Splenopexy for Wandering Spleen; in Saxena AK, Höllwarth ME (eds). Essentials of Pediatric Endoscopic Surgery. Berlin, Heidelberg, Springer Berlin Heidelberg, 2009, [cited 2019 Dec 5], pp 363-367. doi: 10.1007/978-3-540-783879_52.

7. Kristinsson SY, Gridley G, Hoover RN, Check D, Landgren $\mathrm{O}$. Long-term risks after splenectomy among 8,149 cancer-free American veterans: a cohort study with up to 27 years follow-up. Haematologica. 2014; 99: 392-8. doi: 10.3324/haematol.2013.092460.

8. Bonanni P, Grazzini M, Niccolai G, Paolini D, Varone $\mathrm{O}$, Bartoloni A, et al. Recommended vaccinations for asplenic and hyposplenic adult patients. Hum Vaccines Immunother. 2017; 13: 359-68. doi: 10.1080/21645515.2017.1264797.

9. Kim S-C, Kim D-Y, Kim I-K. Avulsion of wandering spleen after traumatic torsion. J Pediatr Surg. 2003; 38: 622-3. doi: 10.1053/jpsu.2003.50136. 